\title{
Minimized extracorporeal circulation is improving outcome of coronary artery bypass surgery in the elderly
}

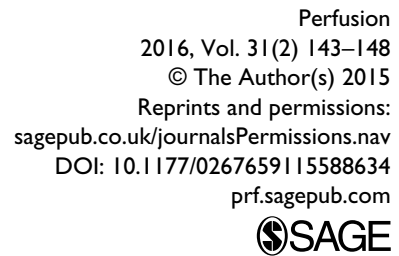

(S)AGE

\author{
Miriam Freundt, Michael Ried, Alois Philipp, Claudius Diez, \\ Philipp Kolat, Stephan W Hirt, Christof Schmid and Assad Haneya
}

\begin{abstract}
Advanced age is a known risk factor for morbidity and mortality after coronary artery bypass grafting (CABG). Minimized extracorporeal circulation (MECC) has been shown to reduce the negative effects associated with conventional extracorporeal circulation (CECC). This trial assesses the impact of MECC on the outcome of elderly patients undergoing $C A B G$. Eight hundred and seventy-five patients (mean age 78.35 years) underwent isolated CABG using CECC $(n=345)$ or MECC $(n=530)$. The MECC group had a significantly shorter extracorporeal circulation time (ECCT), cross-clamp time and reperfusion time and lower transfusion needs. Postoperatively, these patients required significantly less inotropic support, fewer blood transfusions, less postoperative hemodialysis and developed less delirium compared to CECC patients. In the MECC group, intensive care unit (ICU) stay was significantly shorter and 30-day mortality was significantly reduced [ $2.6 \%$ versus $7.8 \% ; p<0.00 \mathrm{I}]$. In conclusion, $M E C C$ improves outcome in elderly patients undergoing CABG surgery.
\end{abstract}

\section{Keywords}

minimized extracorporeal circulation; coronary artery bypass surgery; postoperative outcome; elderly; conventional extracorporeal circulation

\section{Introduction}

Population aging is a global phenomenon and many first world countries will face a situation where, within the next twenty years, the largest population cohort will be those over $65 .{ }^{1}$ Reportedly, in $2009,50.8 \%$ of individuals undergoing cardiac surgery with cardiopulmonary bypass $(\mathrm{CPB})$ were over 69 years of age and $11.8 \%$ were older than 80 years. ${ }^{2}$ Age is an established independent risk factor for morbidity, mortality and adverse events following coronary artery bypass grafting (CABG) and a review of the Society of Thoracic Surgeons (STS) database showed increased mortality of nonagenarians undergoing cardiac surgery. ${ }^{3}$

Conventional CABG using CECC can be associated with severe complications, such as stroke, peripheral embolization, acute kidney injury and systemic inflammatory response syndrome (SIRS). ${ }^{4}$ In open-heart surgery, the main etiology for SIRS is blood cell contact with the artificial bypass surfaces, such as the oxygenator membranes and CPB tubing.., 6 SIRS is characterized by complement activation and the release of cytokines and vasoactive peptides, which can, subsequently, lead to complications, including cardiac arrhythmias, coagulopathy or thromboembolism. ${ }^{4}$ The MECC system was engineered in 2000; details have been previously published by our group. ${ }^{7}$ The system was designed to provide adequate tissue perfusion with much shorter tubing and a more biocompatible pump-oxygenator circuit than conventional CECC. Previous studies indicate that there is a significant reduction in CPB-associated side effects, including SIRS, hemolysis, hemodilution and coagulopathy. ${ }^{8,9}$ Data also indicate a reduced need for postoperative transfusions, less renal and myocardial damage, shorter length of stay and a lower incidence of sternal wound infections, with an explicit benefit in high-risk

\footnotetext{
Dept. of Cardiothoracic Surgery, University Medical Center Regensburg, Franz-Josef-Strauss-Allee II, D-93053 Regensburg, Germany

Corresponding author:

Miriam Freundt, Saint Joseph Hospital, Internal Medicine Residency

Program, 1835 Franklin Street, Denver, CO, 802I8, USA.

Email: miriamfreundt@me.com
} 
Table I. Demographic data and preoperative risk factors.

\begin{tabular}{|c|c|c|c|}
\hline & CECC & MECC & $\mathrm{P}$-value \\
\hline No. of patients & $345(39.4 \%)$ & $530(60.6 \%)$ & \\
\hline Mean age, (range) years & $78.2 \pm 2.9(75-91)$ & $78.5 \pm 3.0(75-89)$ & 0.14 \\
\hline Gender, male & $243(70.4 \%)$ & $365(68.9 \%)$ & 0.65 \\
\hline Body mass index, $\mathrm{kg} / \mathrm{m}^{2}$ & $28.2 \pm 4.2$ & $27.8 \pm 3.8$ & 0.15 \\
\hline EuroScore, \% & $10.8 \pm 8.3$ & $10.2 \pm 7.8$ & 0.28 \\
\hline Ejection fraction, \% & $57 \pm 15$ & $58 \pm 14$ & 0.32 \\
\hline Three vessel disease & $287(83.2 \%)$ & $423(79.8 \%)$ & 0.22 \\
\hline \multicolumn{4}{|l|}{ Urgency of surgery } \\
\hline Elective & $168(48.7 \%)$ & $245(46.2 \%)$ & 0.49 \\
\hline Urgent/Emergent & $\mid 77(5 \mathrm{I} .3 \%)$ & $285(52.8 \%)$ & 0.49 \\
\hline Acute myocardial infarction & $53(15.4 \%)$ & $86(16.2 \%)$ & 0.78 \\
\hline Previous cardiac surgery & $25(7.2 \%)$ & $42(7.9 \%)$ & 0.79 \\
\hline Diabetes mellitus & I I 4 (33.0\%) & $154(29.1 \%)$ & 0.23 \\
\hline Peripheral vascular disease & 71 (20.6\%) & $93(17.5)$ & 0.29 \\
\hline Arterial hypertension & $259(75.1 \%)$ & $416(78.5 \%)$ & 0.25 \\
\hline Atrial fibrillation & $34(9.9 \%)$ & $36(6.8 \%)$ & 0.13 \\
\hline Chronic obstructive pulmonary disease & $4 I(11.9 \%)$ & $44(8.3 \%)$ & 0.10 \\
\hline
\end{tabular}

patients, like those suffering diabetes mellitus (DM) and individuals with reduced ejection fraction or chronic kidney disease (CKD). ${ }^{10-13}$ However, limited data are currently available regarding elderly patients undergoing CABG on MECC. Therefore, the purpose of this retrospective study was to evaluate the impact of MECC on the early outcome of elderly patients undergoing CABG.

\section{Methods}

We reviewed our database of 3909 cases for elderly patients who had undergone elective, emergent or urgent isolated CABG surgery between January 2005 and December 2012 in our institution. Eight hundred and seventy-five patients (aged $\geqslant 75$ years, mean age $78.35 \pm$ 2.95 years $)$ underwent CECC $(n=345 ; 39.4 \%)$ or MECC ( $\mathrm{n}=530 ; 60.6 \%)$ and were included. Exclusion criteria were heparin-induced thrombocytopenia and combined procedure (CABG plus valve or aortic surgery). Baseline characteristics of the population are shown in Table 1. MECC was not implemented in patients with significant aortic insufficiency or a body mass index (BMI) $>30 \mathrm{~kg} / \mathrm{m}^{2}$. Data analysis was performed retrospectively from anonymized data, routinely collected for the German Federal Office of Quality Assurance in Cardiac Surgery. According to the guidelines in our institution, ethics committee approval was not needed for retrospective analysis of anonymized data.

\section{Perfusion procedure with CECC}

We used an extracorporeal circuit consisting of a diffusion membrane oxygenator (Quadrox 2000, Maquet Cardiopulmonary, Rastatt, Germany) and a non- pulsatile roller pump, established for a blood flow of $2.5 \mathrm{~L} / \mathrm{min} / \mathrm{m}^{2}$ (HL 30, Maquet Cardiopulmonary ${ }^{\circledR}$ ), a two-stage cannula (39-50 Fr) (Stöckert', Munich, Germany), draining venous blood from the right atrium, and a 22 Fr aortic cannula (Maquet Cardiopulmonary) for the distal ascending aorta. Lost blood was collected in an open cardiotomy reservoir and transfused back to the patient. The total surface area was $>12 \mathrm{~m}^{2}$ and the system requires a priming volume of $1200 \mathrm{ml}$, including $5000 \mathrm{IU}$ heparin. In addition, $350 \mathrm{IU} / \mathrm{kg}$ heparin were given as a bolus after harvesting the bypass grafts, with a goal activated clotting time (ACT) of $>450$ seconds. We used a single shot of crystalloid cardioplegia (Bretschneider HKT, Franz-Koehler-Chemie", AlsbachHaenlein, Germany) or Calafiore blood cardioplegia to initiate cardiac arrest, depending on the surgeon's preference.

\section{Perfusion procedure with MECC}

We used a closed-loop MECC circuit without air-blood contact. It consisted of a diffusion membrane oxygenator (Quadrox D, Maquet Cardiopulmonary'), a centrifugal pump (RotaFlow, Maquet Cardiopulmonary'), a table line (3/8", 2 x $90 \mathrm{~cm})$, a venous two-stage cannula (32-40 $\mathrm{Fr}$ ), an aortic cannula (21 Fr) and a 1000-ml infusion bag with sodium chloride. The total surface area was $<3 \mathrm{~m}^{2}$ and less than $600 \mathrm{ml}$ of priming volume were required without the addition of heparin. The tubing was prepared before surgery, pre-connected and comes completely coated with heparin. Therefore, an intraoperative ACT of 250-300 sec was sufficient and only $150 \mathrm{IU} / \mathrm{kg}$ of heparin were administered before cross-clamping. In all cases, warm Calafiore blood cardioplegia was used. A cell-saving device (Cell Saver, Hemonetics GmbH, 
Munich, Germany) was used to salvage intraoperative blood loss.

\section{Surgical Procedure}

The same group of experienced senior surgeons, trained and capable to use both MECC and CECC, performed all cases. The decision for MECC or CECC was based on the discretion of the operating surgeon. In all elective cases, platelet inhibitors or anticoagulation were stopped seven days prior to surgery, if possible. Heparin was stopped six hours preoperatively. All patients underwent generalized anesthesia with propofol, fentanyl and pancuronium per standardized institutional protocol. In all cases, a median sternotomy was performed. The internal mammary artery (IMA) and either the radial artery or the saphenous vein were harvested prior to the institution of CPB. In both groups, a brief period of hypothermia $\left(34^{\circ} \mathrm{C}\right)$ was established. Distal anastomoses were sutured in cardiac arrest. Reperfusion was performed per protocol and CPB was weaned. The chest was closed with common sternotomy wires. The patients remained sedated and intubated and were transferred to the ICU for standardized postoperative weaning and care. Extracorporeal circulation time (ECCT), crossclamp time and reperfusion time and the number of transfused red blood cell (RBC) units were recorded.

\section{Postoperative course}

Immediately after arrival in the ICU, labs were collected, including complete blood count (CBC), basic metabolic panel (BMP) and lactate and cardiac enzymes (T1), which were repeated 6 hours postoperatively (T2) and on the morning of postoperative day (POD) 1 (T3). After the achievement of hemodynamic stability and normothermia, sedation and ventilator settings were weaned and early extubation was attempted. The patients were then transferred to the step-down unit for mobilization and later to the regular floor for standard postoperative care. All patients were enrolled into cardiac rehab after discharge. The need for inotropic support, re-exploration due to bleeding, hemodialysis (HD) and $\mathrm{RBC}$ transfusion, 48-hr postoperative blood loss, duration of mechanical ventilation, ICU and hospital stay in days, occurrence of atrial fibrillation (AF), delirium, stroke and infection rates (pulmonary and deep sternal wound infections) were monitored. Death of any cause within 30 days postoperatively was recorded and defined as mortality.

\section{Statistical analysis}

We used SPSS 18.0 (SPSS, Chicago, IL) and Stata 10 SE (Stata Corp, College Station, TX). Continuous data are presented as mean $\pm \mathrm{SD}$ or as median with interquartile range when appropriate. Categorical variables are reported as frequency distributions (n) and simple percentages (\%). Multivariate logistic regression analysis for risk factors associated with 30-day mortality was performed. P-values of $<0.05$ were considered statistical significant.

\section{Results}

\section{Baseline characteristics}

Table 1 shows the baseline characteristics and preoperative risk factors of both groups. The MECC group $(n=530$; $60.6 \%)$ was larger than the CECC group ( $\mathrm{n}=345 ; 39.4 \%)$, but no statistically significant differences occurred regarding risk factors, including age, gender, BMI, EuroScore, left ventricular ejection fraction (LVEF), urgency status of surgery, acute infarction, previous surgery, DM, AF, hypertension, chronic obstructive pulmonary disease or peripheral vascular disease.

\section{Intraoperative data}

There were no statistically significant differences in the use of distal anastomoses between the groups. However, in the MECC group, we found a statistically significant shorter ECCT ( $78 \pm 27$ min versus $95 \pm 35$ min; $\mathrm{p}<0.001$ ), cross-clamp time ( $46 \pm 16$ min versus $53 \pm 18 \mathrm{~min}$; $\mathrm{p}<0.001)$ and reperfusion time $(26 \pm 14$ min versus $34 \pm$ $19 \mathrm{~min} ; \mathrm{p}<0.001)$, as well as lower $\mathrm{RBC}$ transfusion needs [ $\mathrm{n}=177$ (33.4\%) versus 225 (65.2\%); $\mathrm{p}<0.001$ ] (Table 2).

\section{Laboratory data}

No significant difference was seen in the baseline labs (T0) between the groups. Postoperatively, the only statistically significant variable was lactate $(\mathrm{mg} / \mathrm{dL})$, which we found significantly lower in the MECC group (T1: $13 \pm 12$ versus $23 \pm 21 ; \mathrm{p}<0.001, \mathrm{~T} 2: 15 \pm 17$ versus $28 \pm 32 ; \mathrm{p}<0.001, \mathrm{~T} 3: 10 \pm 9$ versus $13 \pm 8 ; \mathrm{p}<0.001)$. No differences were observed in $\mathrm{CBC}$ and creatinine (Table 3).

\section{Postoperative course and data}

Table 4 reports the postoperative differences of both groups. Patients in the MECC group required significantly less inotropic support [47 (8.9\% versus $56(16.2 \%)$; $\mathrm{p}<0.001]$, fewer blood transfusions $(1[0-2]$ units versus $2[0-3]$ units; $\mathrm{p}<0.001)$, less postoperative hemodialysis $[\mathrm{n}=12(2.3 \%)$ versus $\mathrm{n}=20(5.8 \%) ; \mathrm{p}=0.009]$ and developed less delirium $[n=17(3.2 \%)$ versus $n=22(6.4 \%)$; $\mathrm{p}=0.03$ ] compared to patients in the CECC group. In addition, in the MECC group, ICU stay was significantly shorter $(2[2-4]$ days versus $3[2-5]$ days; $\mathrm{p}<0.01)$ and 30 -day mortality was significantly reduced $[\mathrm{n}=14$ 
Table 2. Intraoperative course.

\begin{tabular}{lllr}
\hline & CECC $(\mathrm{n}=345)$ & MECC $(\mathrm{n}=530)$ & $\mathrm{P}$-value \\
\hline Use of internal thoracic artery (\%) & $32 I(93.0 \%)$ & $498(93.9 \%)$ & 0.58 \\
Number of distal anastomoses & $2.9 \pm 0.7$ & $3.0 \pm 0.8$ & 0.45 \\
Extracorporeal circulation time, minutes & $95 \pm 35$ & $78 \pm 27$ & $<0.00 I$ \\
Cross-clamp time, minutes & $53 \pm 18$ & $46 \pm 16$ & $<0.00 I$ \\
Reperfusion time, minutes & $34 \pm 19$ & $26 \pm 14$ & $177(33.4 \%)$ \\
Transfusion of red blood cells (\%) & $225(65.2 \%)$ & $0[0-I]$ & $<0.00 I$ \\
Number of packed red blood cells, unit & $I[0-2]$ & & $<0.00 I$ \\
\hline
\end{tabular}

Table 3. Biochemical/serological data.

\begin{tabular}{|c|c|c|c|}
\hline & CECC $(n=345)$ & $\operatorname{MECC}(n=530)$ & $\mathrm{p}$-value \\
\hline \multicolumn{4}{|c|}{ Hemoglobin (mg/dL) } \\
\hline TO & $12.1 \pm 1.9$ & $12.1 \pm 1.6$ & 0.74 \\
\hline $\mathbf{T I}$ & $9.9 \pm 1.0$ & $9.9 \pm 1.9$ & 0.79 \\
\hline T2 & $10.4 \pm 5.2$ & $10.2 \pm 5.2$ & 0.82 \\
\hline T3 & $10.9 \pm 1.8$ & $\mathrm{II} . \mathrm{I} \pm \mathrm{I} .7$ & 0.10 \\
\hline \multicolumn{4}{|c|}{ Platelets (x $\left.10^{3} / \mu L\right)$} \\
\hline TO & $240 \pm 69$ & $250 \pm 87$ & 0.07 \\
\hline $\mathbf{T} \mathbf{I}$ & $152 \pm 52$ & $194 \pm 84$ & 0.30 \\
\hline $\mathbf{T} 2$ & $161 \pm 56$ & $168 \pm 69$ & 0.13 \\
\hline T3 & $217 \pm 67$ & $226 \pm 79$ & 0.08 \\
\hline \multicolumn{4}{|c|}{ 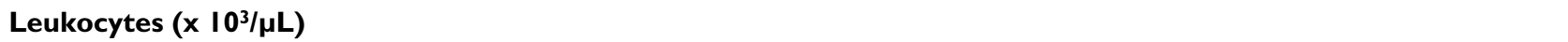 } \\
\hline TO & $8.7 \pm 5.3$ & $8.2 \pm 2.6$ & 0.06 \\
\hline $\mathbf{T} \mathbf{I}$ & $13.8 \pm 14.7$ & $11.8 \pm 9.9$ & 0.02 \\
\hline $\mathbf{T 2}$ & $10.9 \pm 8.9$ & $10.4 \pm 6.6$ & 0.32 \\
\hline T3 & $8.8 \pm 3.6$ & $8.5 \pm 2.9$ & 0.44 \\
\hline \multicolumn{4}{|c|}{ Lactate (mg/dL) } \\
\hline TO & $12 \pm 10$ & $11 \pm 8$ & 0.12 \\
\hline $\mathbf{T I}$ & $23 \pm 21$ & $13 \pm 12$ & $<0.001$ \\
\hline T2 & $28 \pm 32$ & $15 \pm 17$ & $<0.001$ \\
\hline T3 & $13 \pm 8$ & $10 \pm 9$ & $<0.001$ \\
\hline \multicolumn{4}{|c|}{ Creatinine (mg/dL) } \\
\hline TO & $1.3 \pm 0.9$ & $1.2 \pm 0.9$ & 0.21 \\
\hline $\mathbf{T I}$ & $1.3 \pm 1.1$ & $1.3 \pm 1.0$ & 0.73 \\
\hline T2 & $1.3 \pm 0.9$ & $\mathrm{I} . \mathrm{I} \pm 0.5$ & $<0.001$ \\
\hline T3 & $1.4 \pm 0.8$ & $1.3 \pm 0.7$ & 0.10 \\
\hline
\end{tabular}

Blood samples were collected preoperatively (T0), $30 \mathrm{~min}$ after arrival at the intensive care unit (TI), 6 hours after surgery (T2) and at discharge (T3).

(2.6\%) versus $\mathrm{n}=27(7.8 \%) ; \mathrm{p}<0.001]$. No statistically significant difference was found between the groups in 48-hour postoperative drainage output, need for resternotomy due to bleeding, the occurrence of AF, postoperative infections (pulmonary and deep sternal wound infections), stroke and hospital stay (Table 4).

\section{Independent risk factors for 30-day} mortality

In addition, we found that duration of $\mathrm{CPB}$ (Odds ratio 1.03, CI $1.02-1.04, \mathrm{p}<0.001$ ), cross-clamp time (Odds ratio 0.97 , CI $0.94-0.99, \mathrm{p}=0.016$ ) and postoperative $\mathrm{HD}$ (Odds ratio 13.72, CI 5.27 - 35.74, $\mathrm{p}<0.001$ ) were independent risk factors for 30-day mortality. No significance was found for CECC, urgency of surgery or blood transfusion. The results are summarized in Table 5.

\section{Discussion}

In a prior study, patients over 75 years of age were found to have no difference in mortality, myocardial infarction or stroke if they undergo CABG compared to drug-eluting stent (DES) placement, but a significantly higher 
Table 4. Postoperative data.

\begin{tabular}{|c|c|c|c|}
\hline & $\operatorname{CECC~}(n=345)$ & $\operatorname{MECC}(n=530)$ & $\mathrm{P}$-value \\
\hline Inotropic support & $56(16.2 \%)$ & 47 (8.9\%) & $<0.001$ \\
\hline 48-hour drainage loss, $\mathrm{mL}$ & $560[350-850]$ & $550[350-840]$ & 0.65 \\
\hline Re-exploration for bleeding & $10(2.9 \%)$ & $19(3.6 \%)$ & 0.70 \\
\hline Number of packed red blood cells, unit & $2[0-3]$ & $\mathrm{I}[0-2]$ & $<0.001$ \\
\hline Duration of ventilation, hours & $12[9-17]$ & $11[8-15]$ & $<0.01$ \\
\hline Postoperative temporary dialysis & $20(5.8 \%)$ & $12(2.3 \%)$ & 0.009 \\
\hline Postoperative atrial fibrillation & $28(8.1 \%)$ & $58(5.5 \%)$ & 0.13 \\
\hline Pulmonary infection & $20(5.8 \%)$ & $17(3.2 \%)$ & 0.08 \\
\hline Deep sternal wound infection & $23(6.7 \%)$ & $33(6.2 \%)$ & 0.78 \\
\hline Delirium & $22(6.4 \%)$ & 17 (3.2\%) & 0.03 \\
\hline Stroke & $8(2.3 \%)$ & $7(1.3 \%)$ & 0.29 \\
\hline Intensive care unit stay, days & $3[2-5]$ & $2[2-4]$ & $<0.01$ \\
\hline Hospital stay, days & $10[8-12]$ & $9[8-12]$ & 0.45 \\
\hline 30-day mortality & $27(7.8 \%)$ & $14(2.6 \%)$ & $<0.001$ \\
\hline
\end{tabular}

Table 5. Multivariate logistic regression analysis* for risk factors associated with 30 -day mortality.

$*=$ Hosmer-Lemeshow-Test $x^{2}: p=0.309$.

\begin{tabular}{lccc}
\hline & Odds ratio & $95 \%$ Confidence interval & $\mathrm{P}$-value \\
\hline Conventional extracorporeal circulation & 1.75 & $0.78-3.95$ & 0.18 \\
Urgency of surgery & 1.14 & $0.71-1.84$ & 0.59 \\
Extracorporeal circulation time & 1.03 & $1.02-1.04$ & $\mathbf{0 . 0 0 I}$ \\
Cross-clamp time & 0.97 & $0.94-0.99$ & $\mathbf{0 . 0 1 6}$ \\
Transfusion of red blood cell & 1.45 & $0.63-3.36$ & 0.39 \\
Postoperative temporary dialysis & 13.72 & $5.27-35.74$ & $\mathbf{0 . 0 0 I}$ \\
\hline
\end{tabular}

incidence of repeat intervention with DES placement. ${ }^{14}$ Kahn et al. have shown that more frail and elderly patients are undergoing cardiac surgery nowadays than 10 years ago. They have increased mortality and are at increased risk for prolonged ICU stay and adverse events, such as stroke, infections and acute kidney injury (AKI). ${ }^{15}$ In their study, about $60 \%$ were isolated CABG cases, reviewed 2001-2010, and the mortality for patients defined as frail was $14 \%$. Patients included in their study had an average EuroScore of $6 \%$. A review of the STS database for 108 nonagenarians undergoing cardiac surgery from 2002 to 2012 showed a high prevalence of preoperative cerebrovascular disease (23.1\%) and arrhythmia (55.6\%). Overall, nonagenarian mortality was $13 \% .^{3}$ Therefore, it is imperative to find lowerrisk solutions for elderly patients undergoing CABG.

Our study reviewed 875 elderly patients, aged 75-91 years, with a EuroScore of $10.5 \%$, who underwent isolated CABG surgery with either MECC or CECC and found significantly reduced ECCT, cross-clamp and reperfusion time, intra- and postoperative RBC transfusions, need for inotropic support and hemodialysis and stroke and delirium, as well as shorter ICU stay and reduced 30-day mortality in the MECC group. These data are mainly consistent with previously published results of analyses of other high-risk patient populations undergoing $\mathrm{CABG}$. Our group is very experienced with MECC and has demonstrated favorable outcome compared to CECC in several previous publications: MECC had reduced postoperative mortality, length of stay, lower transfusion requirements, less renal and myocardial damage and a lower incidence of sternal wound infections in diabetic patients. ${ }^{10}$ However, in those patients, the predicted perioperative risk according to the EuroScore was lower (4.9\%) than in the elderly population evaluated in this study (10.5\%). Another previous analysis showed that especially high-risk patients of any age with a EuroScore $>10 \%$ had less myocardial damage, lower transfusion rates, less AKI and lower 30-day mortality. ${ }^{9}$ However, this study did not reveal a significant difference in postoperative creatinine levels. Independent risk factors for 30-day mortality in elderly patients were duration of $\mathrm{CPB}$, cross-clamp time and postoperative $\mathrm{HD}$, which were all shorter in the MECC group. A previous analysis of younger patients identified duration of $\mathrm{CPB}$, cross-clamp time, AKI and preoperative myocardial infarction as independent risk factors. ${ }^{16}$

These results allow us to suggest that MECC is not only a safe, but also a superior alternative for CABG in elderly patients compared to CECC. 
Possible explanations for better results with MECC have been discussed in detail by prior authors. ${ }^{9,10,17}$ Most likely, the reduction of blood-artificial surface contact and the reduction in priming volume decrease inflammatory response, hemodilution and third spacing. The lower lactate reflects better tissue perfusion and less anaerobic cell metabolism, as shown by our results in elderly as well as other populations. ${ }^{10}$ Other approaches to reduce mortality in elderly patients undergoing $\mathrm{CABG}$ are off-pump coronary artery bypass grafting $(\mathrm{OPCAB})$ and minimally invasive cardiac surgery CABG (MICS-CABG). Both have been widely discussed in the literature and are controversial due to limited exposure of the surgical field, less surgical control and acuity and hemodynamic instability during exposure of the posterior wall. However, the GOPCABE study group found that, in patients 75 years of age or older, there was no significant difference between on-pump and off-pump CABG with regard to death, stroke, myocardial infarction, repeat revascularization or new renal-replacement therapy within 30 days and 12 months after surgery. ${ }^{18}$ Even further, van Boven et al. reported that, in elderly patients, MECC was associated with an improved early postoperative respiratory performance and lower transfusion rates compared to CECC or OPCAB. ${ }^{19}$

Our study has some limitations. This was a retrospective database analysis from one single center. The groups were not randomized or blinded and selection bias might contribute. Confounding factors might have contributed and influenced the outcome.

Concluding from our results, MECC is superior to CECC in 75-91-year-old patients undergoing CABG surgery. Especially, reduced ECCT, 30-day mortality and lengths of ICU stay, as well as lower transfusion rates, encourage the use of MECC in elderly patients.

\section{Declaration of Conflicting Interest}

The authors declare that there is no conflict of interest.

\section{Funding}

This research received no specific grant from any funding agency in the public, commercial or not-for-profit sectors.

\section{References}

1. Lutz W, Sanderson W, Scherbov S. The coming acceleration of global population ageing. Nature 2008; 451: 716-719.

2. Gummert JF, Funkat A, Beckmann A, et al. Cardiac surgery in Germany during 2009. A report on behalf of the German Society for Thoracic and Cardiovascular Surgery. Thorac Cardiovasc Surg 2010; 58: 379-386.

3. Davis JP, LaPar DJ, Crosby IK, et al. Nonogenarians undergoing cardiac surgery. J Card Surg 2014; 29: 600604.
4. Royston D. The inflammatory response and extracorporeal circulation. J Cardiothorac Vasc Anesth 1997; 11: 341-354.

5. Asimakopoulos G, Taylor KM. Effects of cardiopulmonary bypass on leukocyte and endothelial adhesion molecules. Ann Thorac Surg 1998; 66: 2135-2144.

6. Larmann J, Theilmeier G. Inflammatory response to cardiac surgery: cardiopulmonary bypass versus noncardiopulmonary bypass surgery. Best Pract Res Clin Anaesthesiol 2004; 18: 425-438. Review.

7. Philipp A, Foltan M, Thrum A, Birnbaum D. MECC - a minimal ECC system for coronary artery bypass procedures J Extra Corpor Technol 2002; 34: A215.

8. Fromes Y, Gaillard D, Ponzio O, et al. Reduction of the inflammatory response following coronary bypass grafting with total minimal extracorporeal circulation. Eur $J$ Cardio-thorac Surg 2002; 22: 527-533.

9. Haneya A, Philipp A, Schmid C, et al. Minimised versus conventional cardiopulmonary bypass: outcome of highrisk patients. Eur J Cardio-thorac Surg 2009; 36: 844-848.

10. Haneya A, Puehler T, Philipp A, et al. Coronary artery bypass grafting in patients with type 2 diabetes mellitus: a comparison between minimized and conventional extracorporeal circulation. ASAIO J 2011; 57: 501-506.

11. Munos E, Calderon J, Pillois X, et al. Beating-heart coronary artery bypass surgery with the help of mini extracorporeal circulation for very high-risk patients. Perfusion 2011; 26: 123-131.

12. Puehler T, Haneya A, Philipp A, et al. Minimized extracorporeal circulation in coronary artery bypass surgery is equivalent to standard extracorporeal circulation in patients with reduced left ventricular function. Thorac Cardiovasc Surg 2010; 58: 204-209.

13. Diez C, Haneya A, Brunger F, et al. Minimized extracorporeal circulation cannot prevent acute kidney injury but attenuates early renal dysfunction after coronary bypass grafting. ASAIO J 2009; 55: 602-607.

14. Hannan EL, Zhong Y, Berger PB, et al. Comparison of intermediate-term outcomes of coronary artery bypass grafting versus drug-eluting stents for patients $\geqslant 75$ years of age. Am J Cardiol 2014; 113: 803-808.

15. Buth KJ, Gainer RA, Legare JF, Hirsch GM. The changing face of cardiac surgery: practice patterns and outcomes 2001-2010. Can J Cardiol 2014; 30: 224-230.

16. Kolat P, Ried M, Haneya A, et al. Impact of age on early outcome after coronary bypass graft surgery using minimized versus conventional extracorporeal circulation. $J$ Cardiothorac Surg 2014; 9: 143.

17. Haneya A, Philipp A, Diez C, et al. Comparison of two different minimized extracorporeal circulation systems: hematological effects after coronary surgery. ASAIO J 2009; 55: 592-597.

18. Diegeler A, Borgermann J, Kappert U, et al. Off-pump versus on-pump coronary-artery bypass grafting in elderly patients. NEJM 2013; 368: 1189-1198.

19. van Boven WJ, Gerritsen WB, Driessen AH, van Dongen EP, Klautz RJ, Aarts LP. Minimised closed circuit coronary artery bypass grafting in the elderly is associated with lower levels of organ-specific biomarkers: a prospective randomised study. Eur J Anaesthesiol 2013; 30: 685-694. 